\title{
On the Convergence of Non-Uniform Wavelet Packet Expansions
}

\author{
Sohrab Ali \\ Department of Mathematics, Jamia Millia Islamia, New Delhi -110025, India..
}

\begin{abstract}
In this paper, we study the pointwise convergence of nonuniform wavelet packet expansions and show that such expansions converge pointwise almost everywhere, and more precisely everywhere on the Lebesgue set of the function being expanded. Certain results are obtained in this direction by assuming only that the nonuniform wavelet packets being used be bounded by radial decreasing $L^{1}$-functions.
\end{abstract}

Keywords: Nonuniform multiresolution analysis, Wavelet packets, Radial decreasing $L^{1}$-functions.

\section{Introduction}

A simple, but powerful extension of wavelets and multiresolution analysis is wavelet packets. Wavelet packet functions comprise a rich family of building block functions and are localised in time, but offer more flexibility than wavelets in representing different types of signals. In particular, wavelet packets are better at representing signals that exhibit oscillatory or periodic behavior.

In his paper, Mallat [11] first formulated the remarkable idea of multiresolution analysis (MRA) that deals with a general formlism for construction of an orthogonal basis of wavelet bases. A multiresolution analysis consists of a sequence of embedded closed subspaces $\left\{V_{j}: j \in \mathbb{Z}\right\}$ for approximating $L^{2}(\mathbb{R})$ functions (see [7]). The notions of MRA and wavelets were generalised to many different settings. One can replace the dilation factor 2 by an integer $M \geq 2$. In general, in higher dimensions, it can be replaced by a dilation matrix $A$, in which case the number of wavelets required is $|\operatorname{det} A|-1$. But in all these cases, the translation set is always a group. In the two papers [4,5], Gabardo and Nashed considered a generalisation of Mallat's [11] celebrated theory of MRA, in which the translation set acting on the scaling function associated with the MRA to generate the subspace $V_{0}$ is no longer a group, but is the union of $\mathbb{Z}$ and a translate of $\mathbb{Z}$. More precisely, this set is of the form $\{0, r / N\}+2 \mathbb{Z}$, where $N \geq 1$ is an integer, $1 \leq r \leq 2 N-1, r$ is an odd integer relatively prime to $N$. They call this a nonuniform multiresolution analysis (NUMRA). Later on, Behera [2] constructed the associated nonuniform wavelet packets for this nonuniform multiresolution analysis. For basic construction of nonuniform wavelets and associated nonuniform wavelet packets, we refer to [2, 4-6].

The problem of convergence of the wavelet series has been studied by Meyer [12], Walter [15, 16], and Kelly et al. $[9,10]$. Meyer was amongst the first to study convergence results for wavelet expansions. He has shown that the regular wavelet expansions converge in $L^{p}, 1 \leq p<\infty$ and also in $L^{\infty}$ for expansions of uniformly continuous functions, the expansion of continuous functions converge everywhere. The results in [12] were based on the assumption of so called regularity for the basic wavelets and their derivatives. In addition, Walter $[15,16]$ established pointwise convergence results for regular wavelet expansions of continuous functions. Kelly et al. $[9,10]$ have extended and obtained results analogous to those obtained by Carleson [3] and Hunt [8] for the Fourier series. In contrast, the results in $[9,10]$ assumed only that the wavelets being used be bounded by radial decreasing $L^{1}$-functions. In [10], it is shown that the wavelet expansions of a function belonging to $L^{p}$ converges pointwise everywhere on the Lebesgue set of a given function, for $1 \leq p<\infty$. Recently, Ahmad and Kumar [1] have extended the results of Kelly et al. $[9,10]$ to the stationary wavelet packets and have shown that wavelet packets expansion of any $L^{p}$-function $(1 \leq p \leq \infty)$ converges pointwise almost everywhere under certain conditions. On the other hand, Nielson $[13,14]$ introduced the non-stationary wavelet packets generated by the Haar filters which he called them Walsh-type wavelet packets for which the same type of $L^{p}$-convergence results hold.

Motivated and inspired by the importance of nonuniform wavelet packets, in the present paper, we study the pointwise convergence of nonuniform wavelet packet series by assuming that the nonuniform wavelet packets being used be bounded by radial decreasing $L^{1}$-functions.

\section{Preliminaries}

Definition 2.1. Let $N$ be an integer, $N \geq 1$, and $\Lambda=\{0, r / N\}+2 \mathbb{Z}$, where $r$ is an odd integer relatively prime to $N$ with $1 \leq r \leq 2 N-1$. A sequence $\left\{V_{j}: j \in \mathbb{Z}\right\}$ of closed subspaces of $L^{2}(\mathbb{R})$ is called a nonuniform multiresolution analysis (NUMRA) associated with $\Lambda$ if the following conditions are satisfied:

(2.1) $\quad V_{j} \subset V_{j+1}$ for all $j \in \mathbb{Z}$,

(2.2) $\cup_{j \in \mathbb{Z}} V_{j}$ is dense in $L^{2}(\mathbb{R})$ and $\bigcap_{j \in \mathbb{Z}} V_{j}=\{0\}$, 
(2.3) $f \in V_{j}$ if and only if $f(2 N.) \in V_{j+1}$,

(2.4) There exists a function $\varphi$ in $V_{0}$ such that $\{\varphi(.-\lambda): \lambda \in \Lambda\}$ is an orthonormal basis of $V_{0}$.

The function $\varphi$ whose existence is asserted in (2.4) is called a scaling function of the given NUMRA.

It is worth noting that, when $N=1$, one recovers from the definition above the standard definition of a onedimensional multiresolution analysis with dilation factor equal to 2 . When, $N>1$, the dilation factor of $2 N$ ensures that $2 N \Lambda \subset 2 \mathbb{Z} \subset \Lambda$. Equation (2.3) implies that

where $\sum_{\lambda \in \Lambda}\left|a_{\lambda}\right|^{2}<\infty$.

$$
\varphi\left(\frac{x}{2 N}\right)=\sum_{\lambda \in \Lambda} a_{\lambda} \varphi(x-\lambda)
$$

Now, we consider $W_{0}$ the orthogonal complement of $V_{0}$ on $V_{1}$, i.e.

$$
V_{1}=V_{0} \oplus W_{0} \text {. }
$$

If $\psi_{1}, \psi_{2}, \ldots, \psi_{2 N-1}$ are the functions in $W_{0}$, then for $\ell=0,1, \ldots, 2 N-1$, there exist sequences $\left\{a_{\lambda}^{\ell}\right\}_{\lambda \in \Lambda}$ satisfying $\sum_{\lambda \in \Lambda}\left|a_{\lambda}^{\ell}\right|^{2}<\infty$ such that

Now, consider

$$
\frac{1}{2 N} \psi_{\ell}\left(\frac{x}{2 N}\right)=\sum_{\lambda \in \Lambda} a_{\lambda}^{\ell} \varphi(x-\lambda) .
$$

where the functions $m_{\ell}(\xi)=\sum_{\lambda \in \Lambda} a_{\lambda}^{\ell} e^{-2 \pi i \lambda \xi}$ are locally $L^{2}$. Since $\Lambda=\{0, r / N\}+2 \mathbb{Z}$,

we can write that

$$
m_{\ell}(\xi)=m_{\ell}^{1}(\xi)+e^{-2 \pi i \xi r / N} m_{\ell}^{2}(\xi), \quad \ell=0,1, \ldots, 2 N-1,
$$

where $m_{\ell}^{1}$ and $m_{\ell}^{2}$ are locally $L^{2}, 1 / 2$-periodic functions.

In this case $\left\{\psi_{1}, \psi_{2}, \ldots, \psi_{2 N-1}\right\}$ is a set of basic wavelets associated with a scaling function $\varphi$. It is easy to show that $\left\{\psi_{\ell}(x-\lambda): 1 \leq \ell \leq 2 N-1\right\}$ is an orthonormal basis of $W_{0}$. An obvious rescaling shows that

$$
\left\{\psi_{\ell, j, \lambda}=(2 N)^{j / 2} \psi_{\ell}\left((2 N)^{j} x-\lambda\right): 1 \leq \ell \leq 2 N-1, \lambda \in \Lambda\right\}
$$

is an orthonormal basis of $W_{j}$. Since $\cup_{j \in \mathbb{Z}} V_{j}$ is dense in $L^{2}(\mathbb{R})$, the collection $\left\{\psi_{\ell, j, \lambda}: j \in \mathbb{Z}, \lambda \in \Lambda, 1 \leq \ell \leq\right.$ $2 N-1$, is an orthonormal basis of $L 2 \mathbb{R}$.

We, now, define $\omega_{n}$ for each integer $n \geq 0$ as follows. Suppose that for $p \geq 0, \omega_{p}$ is already defined. Then, define basic nonuniform wavelet packets $\omega_{q+2 N p}, 0 \leq q \leq 2 N-1$, by

$$
\omega_{q+2 N p}(x)=\sum_{\lambda \in \Lambda}(2 N) a_{\lambda}^{q} \omega_{p}(2 N x-\lambda) .
$$

Clearly, the set $\left\{\omega_{n}(x-\lambda): \lambda \in \Lambda, n=0,1, \ldots\right\}$ is an orthonormal basis of $L^{2}(\mathbb{R})$. Corresponding to some orthonormal scaling function $\varphi=\omega_{0}$, the family of nonuniform wavelet packets $\omega_{n}$ defines a family of subspaces of $L^{2}(\mathbb{R})$ as follows:

(2.10) $\quad U_{j}^{n}=\operatorname{span}\left\{(2 N)^{j / 2} \omega_{n}\left((2 N)^{j} x-\lambda\right): \lambda \in \Lambda\right\} ; j \in \mathbb{Z}, n=0,1,2, \ldots$

Since $\omega_{0}=\varphi$ is the scaling function and $\omega_{n}, 1 \leq n \leq 2 N-1$, are the nonuniform wavelet packets, we observe that

$$
U_{j}^{0}=V_{j}, \quad U_{j}^{1}=W_{j}=\bigoplus_{r=1}^{2 N-1} U_{j}^{r}, \quad j \in \mathbb{Z}
$$

So that the orthogonal decomposition $V_{j+1}=V_{j} \oplus W_{j}$, can be written as

$$
U_{j+1}^{0}=U_{j}^{0} \oplus U_{j}^{1}=\oplus_{r=0}^{2 N-1} U_{j}^{r} .
$$

A generalisation of this result for other values of $n=1,2, \ldots$, can be written as

$$
U_{j+1}^{n}=\oplus_{r=0}^{2 N-1} U_{j}^{r+2 N n}, \quad j \in \mathbb{Z} \text {. }
$$

Lemma 2.2 [2]. If $j \geq 0$, then

$$
\begin{gathered}
W_{j}=\bigoplus_{r=1}^{2 N-1} U_{j}^{r}=\bigoplus_{r=2 N}^{(2 N)^{2}-1} U_{j-1}^{r}=\cdots=\bigoplus_{r=(2 N)^{P}}^{(2 N)^{p+1}-1} U_{j-p,}^{r} \quad p \leq j \\
=\bigoplus_{r=(2 N)^{j}}^{(2 N)^{j+1}-1} U_{0}^{r},
\end{gathered}
$$

where $U_{j}^{n}$ is defined in (2.10). Using this decomposition, we get the nonuniform wavelet packets decomposition of subspaces $W_{j}, j \geq 0$. Therefore, for any function $f \in L^{2}(\mathbb{R})$, we have 


$$
f(x)=\sum_{n=(2 N)^{p}}^{(2 N)^{p+1}-1} \sum_{j \in Z} \sum_{\lambda \in \Lambda} C_{r, n, \lambda} \omega_{r, j, \lambda(x),}
$$

where $r=j-p, p=0$ if $j<0$ and $p=0,1,2, \ldots, j$ if $j \geq 0$; will be a nonuniform wavelet packet expansion of $f$ and $C_{r, n, \lambda}$ the wavelet packet coefficients, defined as

$$
C_{r, n, \lambda}=\left\langle f, \omega_{r, j, \lambda}\right\rangle .
$$

Let $P_{j}$ and $Q_{j}$, respectively be the orthogonal projections onto the spaces $V_{j}$ and $W_{j}$ with the kernels $P_{j}(x, y)$ and $Q_{j}(x, y)$, defined as follows:

$$
P_{j}(x, y)=\sum_{\lambda \in \Lambda} \varphi_{j, \lambda}(x) \overline{\varphi_{j, \lambda}(y)},
$$

where $\varphi_{j, \lambda}(x)=(2 N)^{j / 2} \varphi\left((2 N)^{j} x-\lambda\right)$ and

$$
Q_{j}(x, y)=\sum_{\ell=0}^{2 N-1} \sum_{\lambda \in \Lambda} \psi_{\ell, j, \lambda}(x) \overline{\psi_{\ell, j, \lambda}(y)}
$$

In the light of $V_{j+1}=V_{j} \oplus W_{j}, P_{j}(x, y)$ can be written as

$$
P_{j}(x, y)=\sum_{m<j} Q_{m}(x, y)=\sum_{\ell=0}^{2 N-1} \sum_{m<j} \sum_{\lambda \in \Lambda} \psi_{\ell, j, \lambda}(x) \overline{\psi_{\ell, j, \lambda}(y)} .
$$

Now, we consider a projection $Q_{j}^{n}$ onto $U_{j}^{n}$ with kernel $Q_{j}^{n}(x, y)$ defined as

$$
Q_{j}^{n}(x, y)=\sum_{\lambda \in \Lambda} \omega_{j, n, \lambda}(x) \overline{\omega_{j, n, \lambda}(y)} ; j \in \mathbb{Z}, n=0,1,2, \ldots
$$

where $\omega_{j, n, \lambda}$ are the nonuniform wavelet packets. Thus, we observe that $Q_{j}^{0}=P_{j}$ and $Q_{j}^{1}=Q_{j}$. In the light of Lemma 2.2, $Q_{j}$ can be expressed as

$$
Q_{j}(x, y)=\sum_{n=(2 N)^{P}}^{(2 N)^{p+1}-1} Q_{r}^{n}(x, y)=\sum_{n=(2 N)^{P}}^{(2 N)^{p+1}-1} \sum_{j \in \mathbb{Z}} \sum_{\lambda \in \Lambda} \omega_{r, j, \lambda}(x) \overline{\omega_{r, j, \lambda}(y)},
$$

where $r=j-p, p=0$ if $j>0$ and $p=0,1,2, \ldots, j$ if $j \geq 0$.

Thus eq. (2.15) gives

$$
\begin{gathered}
P_{j}(x, y)=\sum_{m<j} Q_{m}(x, y)=\sum_{m<j} \sum_{n=(2 N)^{P}}^{(2 N)^{p+1}-1} Q_{r}^{n}(x, y) \\
=\sum_{m<j \in \mathbb{Z}} \sum_{n=(2 N)^{P}}^{(2 N)^{p+1}-1} \sum_{\lambda \in \Lambda} \omega_{r, j, \lambda}(x) \overline{\omega_{r, j, \lambda}(y),}
\end{gathered}
$$

where $r=m-p, p=0$ if $m<0$ and $p=0,1,2, \ldots, m$ if $0 \leq m<j$.

Now, we consider a natural operator $S_{j, \lambda}^{\sigma}$ associated with nonuniform wavelet packets $\omega_{n}$ with kernel

$$
S_{j, \lambda}^{\sigma}(x, y)=\sum_{m<j} \sum_{n=(2 N)^{P}}^{(2 N)^{p+1}} \sum_{\lambda \in \Lambda} \omega_{r, j, \lambda}(x) \overline{\omega_{r, j, \lambda}(y)}+R_{j, \lambda}^{\sigma}(x, y),
$$

where $r=m-p, p=0$ if $m<0$ and $p=0,1,2, \ldots, m$ if $0 \leq m<j$,

$$
R_{j, \lambda}^{\sigma}(x, y)=\sum_{m=1}^{\lambda} \omega_{j, n, \sigma(m)}(x) \overline{\omega_{j, n, \sigma(m)}(y),}
$$

with $r=j-p, p=0$ if $j<0$ and $p=0,1,2, \ldots j$ if $j \geq 0, j \in \mathbb{Z}, \lambda \in \Lambda$ and $\sigma$ is a permutation of $\mathbb{Z}$. This operator is a partial sum of nonuniform wavelet packet expansion of $f$.

Definition 2.3 [7]. For a function $f$ defined on $\mathbb{R}$, we say that a bounded function $H:[0, \infty) \rightarrow \mathbb{R}^{+}$is a radial decreasing $L^{1}$-majorant of $f$ if $\mid F(X) \leq H(|x|)$ and $H$ satisfies the following conditions:

(i) $H \in L^{1}[0, \infty)$,

(ii) $H$ is decreasing,

(iii) $H(0)<\infty$. 
Definition 2.4 [7]. The point $x \in \mathbb{R}$ is said to be a Lebesgue point of a function $f$ on $\mathbb{R}$ if $f$ is integrable in some neighbourhood of $x$ and

$$
\lim _{\varepsilon \rightarrow 0} \frac{1}{V\left(B_{\varepsilon}\right)} \int_{B_{\varepsilon}}|f(x)-f(x+y)| d y=0,
$$

where $B_{\varepsilon}$ denotes the ball of radius $\varepsilon$ about the origin and $V$ denotes volume.

Lemma 2.5 [7]. Let $H$ be the function on $[0, \infty)$ satisfying the conditions of $(2.20)$. Then

$$
\sum_{\lambda \in \Lambda} H(|x-\lambda|) H(|y-\lambda|) \leq C H\left(\frac{|x-y|}{2 N}\right), \quad \text { for all } x, y \in \mathbb{R},
$$

where $C$ is a constant depending on $H$.

\section{Convergence Results}

Let $\omega_{n}, n=0,1,2, \ldots$ be the nonuniform wavelet packets associated with the increasing sequence of subspaces $\left\{V_{j}\right\}_{j \in \mathbb{Z}}$, then the orthogonal projections of $L^{2}(\mathbb{R})$ onto $V_{j}$ are given by

$$
P_{j} f(x)=\sum_{m<j} \sum_{n=(2 N)^{P}}^{(2 N)^{p+1}} \sum_{\lambda \in \Lambda}\left\langle f, \omega_{r, j, \lambda}\right\rangle \omega_{r, j, \lambda}(x), \text { for all } f \in L^{2}(\mathbb{R}),
$$

where $r=m-p, p=0$ if $m<0$ and $p=0,1,2, \ldots, m$ if $0 \leq m<j$.

We can also consider the projections $Q_{j}^{n}$ from $L^{2}(\mathbb{R})$ onto $U_{j}^{n}$ given by

$$
Q_{j}^{n} f(x)=\sum_{\lambda \in \Lambda}\left\langle f, \omega_{r, j, \lambda}\right\rangle \omega_{r, j, \lambda}(x), \quad \text { for } f \in L^{2}(\mathbb{R}) .
$$

There is also a natural operator, associated with a nonuniform wavelet packet $\omega_{n}$ given by

$$
\left(S_{j, \lambda}^{\sigma} f\right)(x)=\sum_{m<j} \sum_{n=(2 N)^{P}} \sum_{\lambda \in \Lambda}\left\langle f, \omega_{r, j, \lambda}\right\rangle \omega_{r, j, \lambda}(x)+\left(R_{j, \lambda}^{\sigma} f\right)(x),
$$

where $r=m-p, p=0$ if $m<0$ and $p=0,1,2, \ldots, m$ if $0 \leq m<j$,

$$
\left(R_{j, \lambda}^{\sigma} f\right)(x)=\sum_{m=1}^{\lambda} \sum_{n=(2 N)^{P}}^{(2 N)^{p+1}-1}\left\langle f, \omega_{r, n, \sigma(m)}\right\rangle \omega_{r, n, \sigma(m)}(x),
$$

with $r=j-p, p=0$ if $j<0$ and $p=0,1,2, \ldots j$ if $j \geq 0, f \in L^{2}(\mathbb{R})$ and $\sigma$ is any permutation of $\mathbb{Z}$. This operator has a partial sum of nonuniform wavelet packet expansion of $f$. Since

$$
V_{j}=U_{j}^{0}=\bigoplus_{m<j} \bigoplus_{n=(2 N)^{P}}^{(2 N)^{p+1}-1} U_{r}^{n}
$$

where $r=m-p, p=0$ if $m<0$ and $p=0,1,2, \ldots, m$ if $0 \leq m<j$, we have

$$
\left(S_{j, \lambda}^{\sigma} f\right)(x)=P_{j} f(x)+\sum_{m=1}^{\lambda} \sum_{n=(2 N)^{P}}^{(2 N)^{p+1}-1}\left\langle f, \omega_{r, n, \sigma(m)}\right\rangle \omega_{r, n, \sigma(m)}(x),
$$

where $r=j-p, p=0$ if $j<0$ and $p=0,1,2, \ldots, j$ if $j \geq 0$.

The above definition makes sense for any $f \in L^{2}(\mathbb{R})$. Writing $\left\langle f, \omega_{r, n, m}\right\rangle$ as an integral and interchanging the order of summation and integration, we obtain

$$
\left(S_{j, \lambda}^{\sigma} f\right)(x)=\int_{-\infty}^{\infty}(2 N)^{j} P_{0}\left((2 N)^{j} x,(2 N)^{j} y\right) f(y) d y+\int_{-\infty}^{\infty}(2 N)^{j} Q_{0}^{\sigma, \lambda}\left((2 N)^{j} x,(2 N)^{j} y\right) f(y) d y,
$$

where

$$
\left.Q_{0}^{\sigma, \lambda}(x, y)=\sum_{m=1}^{\lambda} \omega_{n}(x-\sigma(m)) \overline{\omega_{n} y-\sigma(m)}\right), \quad \text { where } n=1
$$

and

$$
P_{0}(x, y)=\sum_{j<0} \sum_{\lambda \in \Lambda} \omega_{j, n, \lambda}(x) \overline{\omega_{j, n, \lambda}(y)}, \quad \text { where } n=1
$$




$$
=\sum_{\lambda \in \Lambda} \omega_{0}(x-\lambda) \overline{\omega_{0}(y-\lambda),} \quad \text { as } \omega_{0}=\varphi .
$$

Now, we consider an operator $T_{j}$ defined as

$$
\left(T_{j} f\right)(x) \int_{\mathbb{R}}(2 N)^{j} P_{0}\left((2 N)^{j} x,(2 N)^{j} y\right)\{f(y)-f(x)\} d y,
$$

where

$H$ satisfies (2.20) and $C$ is a constant.

$$
\left|P_{0}(x, y)\right| \leq C H\left(\frac{|x-y|}{2 N}\right)
$$

Theorem 3.1. Let $\left\{T_{j}: j \in \mathbb{Z}\right\}$ be the family of operators defined by (3.8). If $f \in L^{P}(\mathbb{R}), 1 \leq p \leq \infty$, then $\lim _{j \rightarrow \infty} T_{j} f(x)=0$ for every $x$ in the Lebesgue set of $f$.

Proof. From (3.8), we have

$$
\begin{gathered}
\left|T_{j} f(x)\right| \leq \int_{\mathbb{R}}(2 N)^{j}\left|P_{0}\left((2 N)^{j} x,(2 N)^{j} y\right)\right||f(y)-f(x)| d y \\
\leq C \int_{\mathbb{R}}(2 N)^{j} H\left((2 N)^{j-1}|x-y|\right)|f(y)-f(x)| d y \\
\quad=C \int_{\mathbb{R}}(2 N)^{j} H\left((2 N)^{j-1}|t|\right)|f(x-t)-f(x)| d t .
\end{gathered}
$$

If $x$ is a point in the Lebesgue set of $f$ and $\delta>0$, there exists any $\eta>0$ such that (replacing $y$ by $(x-t))$

$$
\frac{1}{s} \int_{|t| \leq s}|f(x-t)-f(x)| d t \leq \delta, \quad 0<s \leq \eta .
$$

Thus

$$
\begin{aligned}
C^{-1}\left|T_{j} f(x)\right| & \leq \int_{|t|<\eta}(2 N)^{j} H\left((2 N)^{j-1}|t|\right)|f(x-t)-f(x)| d t \\
& +\int_{|t| \geq \eta}(2 N)^{j} H\left((2 N)^{j-1}|t|\right)|f(x-t)-f(x)| d t \\
& =I+I I .
\end{aligned}
$$

But $H(|x|)$ decreases to zero as $|x| \rightarrow \infty$ and $H \in L^{1}([0, \infty))$. Therefore

$$
s H(s) \leq \int_{\frac{s}{2 N} \leq|x| \leq s} H(|x|) d x \rightarrow 0 \text { as } s \rightarrow \infty \text {. }
$$

Further, since $H$ is continuous at zero, $s H(s) \rightarrow 0$ as $s \rightarrow 0$. Let

From relation (3.9), we deduce that

$$
g(s)=|f(x-s)-f(x)| \text { and } G(s)=\int_{0}^{s} g(u) d u .
$$

(3.10)

$$
G(s) \leq s \delta \text { when } 0 \leq s \leq \eta
$$

On integrating by parts, we obtain

$$
\begin{gathered}
\quad I=\int_{|t|<\eta}(2 N)^{j} H\left((2 N)^{j-1}|t|\right)|f(x-t)-f(x)| d t \\
=(2 N) \int_{0}^{\eta}(2 N)^{j} H\left((2 N)^{j-1} s\right) g(s) d s \\
=\left.(2 N) G(s)(2 N)^{j} H\left((2 N)^{j-1} s\right)\right|_{0} ^{\eta}-(2 N) \int_{0}^{\eta} G(s)(2 N)^{j}(2 N)^{j-1} H^{\prime}\left((2 N)^{j-1} s\right) d s \\
\left.\leq\left.(2 N)^{j} s \delta(2 N)^{j} H\left((2 N)^{j-1} s\right)\right|_{0} ^{\eta}-(2 N) \int_{0}^{(2 N)^{j} \eta} G(2 N)^{-j} s\right) d s H^{\prime}\left(\frac{s}{2 N}\right) d s .
\end{gathered}
$$

Since $H$ is decreasing, $H^{\prime}\left(\frac{s}{2 N}\right)$ is negative by (3.10) and the boundedness of $s H(s)$, we obtain

$$
\begin{aligned}
& I \leq C \delta-(2 N)^{j} \delta \int_{0}^{(2 N)^{j} \eta}(2 N)^{-j} s(2 N)^{j-1} H^{\prime}\left(\frac{s}{2 N}\right) d s \\
& =C \delta-\left.\delta s(2 N)^{j} H\left(\frac{s}{2 N}\right)\right|_{0} ^{(2 N)^{j} \eta} \delta \int_{0}^{(2 N)^{j} \eta}(2 N)^{j} H\left(\frac{s}{2 N}\right) d s \\
& =C \delta-(2 N)^{j} \delta A H\left(\frac{A}{2 N}\right)+(2 N)^{j} \delta \int_{0}^{\frac{A}{2 N}}(2 N)^{j} H(v) d v,
\end{aligned}
$$


where $A=(2 N)^{j} \eta$ and $s=(2 N)^{j} v$. As $j \rightarrow \infty$ (or equivalently, as $A \rightarrow \infty$ ) the last expression increases to $(2 N)^{2}\|H\|_{L^{1}(\mathbb{R})}$. This gives the estimate

$$
I \leq C \delta+(2 N)^{2}\|H\|_{L^{1}(\mathbb{R})} \delta=a \delta .
$$

Clearly, the constant $a$ depends on $H$ only. In order to estimate $I I$, let $x_{n}$ be the characteristic function of the set $\{t \in \mathbb{R}:|t| \geq \eta\}$. Let $q$ denote the conjugate exponent to $p$, that is $\frac{1}{p}+\frac{1}{q}=1$. By using Hölder's inequality, we get

$$
\begin{aligned}
I I & \leq\|f\|_{L^{p}(\mathbb{R})}\left(\int_{\mathbb{R}}\left|x_{\eta}(t)(2 N)^{j} H\left((2 N)^{j-1}|t|\right)\right|^{q} d t\right)^{\frac{1}{q}} \\
& +|f(x)| \int_{\mathbb{R}}\left|x_{\eta}(t)(2 N)^{j} H\left((2 N)^{j-1}|t|\right)\right| d t .
\end{aligned}
$$

But

$$
\int_{\mathbb{R}}\left|x_{\eta}(t)(2 N)^{j} H\left((2 N)^{j-1}|t|\right)\right| d t=(2 N) \int_{|u| \geq(2 N)^{j-1} \eta} H(|u|) d u,
$$

which tends to zero as $j \rightarrow \infty$. Also, the same is true for the first summand of the above inequality. Further

$$
\begin{gathered}
\left(\int_{\mathbb{R}}\left|x_{\eta}(t)(2 N)^{j} H\left((2 N)^{j-1}|t|\right)\right|^{q} d t\right)^{\frac{1}{q}}=\left(\int_{|t| \geq \eta}\left|(2 N)^{j} H\left((2 N)^{j-1}|t|\right)\right|^{\frac{q}{p}}\left|(2 N)^{j} H\left((2 N)^{j-1}|t|\right)\right| d t\right)^{\frac{1}{q}} \\
\leq\left(\sup _{|t| \geq \eta}\left|(2 N)^{j} H\left((2 N)^{j-1}|t|\right)\right|\right)^{\frac{1}{p}}\left(\left|(2 N)^{j} H\left((2 N)^{j-1}|t|\right)\right|\right)^{\frac{1}{q}} \\
\leq C\left(\|H\|_{L^{1}(0,1)}\right)^{\frac{1}{q}}\left(\sup _{|t| \geq \eta}\left|(2 N)^{j} H\left((2 N)^{j-1}|t|\right)\right|\right)^{\frac{1}{p}} .
\end{gathered}
$$

But $s H(s) \rightarrow 0$ as $s \rightarrow \infty$. Therefore

$$
\sup _{|t| \geq \eta}\left|(2 N)^{j} H\left((2 N)^{j-1}|t|\right)\right|=(2 N)^{j} H\left((2 N)^{j-1} \eta\right)
$$

tends to zero as $j \rightarrow \infty$. Hence, choosing $j$ large enough we deduce from (3.12) that $I I$ can be made smaller than $\delta$. This together with inequality (3.11) proves the result.

Theorem 3.2. Suppose that $\omega_{n}$ are nonuniform wavelet packets and $\omega_{n}$ has a radially decreasing $L^{1}$-majorant. If $f \in L^{p}(\mathbb{R}), 1 \leq p \leq \infty$, then

$$
\lim _{j \rightarrow \infty} P_{j} f(x)=f(x) \text { for any } x \text { in the Lebesgue set of } f .
$$

In particular, $\lim _{j \rightarrow \infty} P_{j} f(x)=f(x)$ for almost every $x \in \mathbb{R}$.

Proof. It is easy to show that

$$
\int_{\mathbb{R}} P_{0}\left((2 N)^{j} x,(2 N)^{j} y\right) d y=1
$$

Now

$$
P_{j} f(x)-f(x)=\int_{\mathbb{R}}(2 N)^{j} P_{0}\left((2 N)^{j} x,(2 N)^{j} y\right)\{f(y)-f(x)\} d y=T_{j} f(x) .
$$

On taking limit $j \rightarrow \infty$ and applying Theorem 3.1, we get

for every $x$ in the Lebesgue set of $f$.

$$
\lim _{j \rightarrow \infty} P_{j} f(x)-f(x)=0 \Rightarrow \lim _{j \rightarrow \infty} P_{j} f(x)=f(x)
$$

Theorem 3.3. Let $\omega_{n}, n \geq 0$ be nonuniform wavelet packets having the radial decreasing $L^{1}$-majorant. If $f \in L^{p}(\mathbb{R}), 1 \leq p \leq \infty$, then, for $\lambda \in \Lambda$,

$$
\lim _{j \rightarrow \infty} S_{j, \lambda}^{\sigma} f(x)=f(x) \text { for all } x \text { in the Lebesgue set of } f .
$$

In particular, the partial sums $S_{j, \lambda}^{\sigma} f(x)$ converge to $f(x)$ for almost every $x \in \mathbb{R}$.

Proof. Since

$$
\int_{\mathbb{R}} \omega_{n}(y) d y=\widehat{\omega_{n}}(0)=0
$$


for all $n>0$, by (3.4) we obtain

$$
\left(S_{j, \lambda}^{\sigma} f\right)(x)-f(x)=\left[P_{j} f(x)-f(x)\right]+R_{j, \lambda}^{\sigma} f(x),
$$

where

$$
R_{j, \lambda}^{\sigma} f(x)=\int_{\mathbb{R}}\left\{\sum_{m=1}^{\lambda} \sum_{n=(2 N)^{p}}^{(2 N)^{p+1}-1} \omega_{r, n, \sigma(m)}(x) \overline{\omega_{r, n, \sigma(m)}(y)}\right\}\{f(y)-f(x)\} d y
$$

where $r=m-p, p=0$ if $j<0$ and $p=0,1,2, \ldots, j$ if $j \geq 0$. Thus

Hence

$$
R_{j, \lambda}^{\sigma} f(x)=\int_{\mathbb{R}}(2 N)^{j} Q_{0}^{\sigma, \lambda}\left((2 N)^{j} x,(2 N)^{j} y\right)\{f(y)-f(x)\} d y .
$$

$$
\left(s_{j, \lambda}^{\sigma} f\right)(x)-f(x)=\left[P_{j} f(x)-f(x)\right]+\int_{\mathbb{R}}(2 N)^{j} Q_{0}^{\sigma, \lambda}\left((2 N)^{j} x,(2 N)^{j} y\right)\{f(y)-f(x)\} d y .
$$

But by Theorem 3.2, $\lim _{j \rightarrow \infty}\left(P_{j} f(x)-f(x)\right)=0$. Therefore, by Lemma 2.5

$$
\left|Q_{0}^{\sigma, \lambda}(x, y)\right| \leq C H\left(\frac{|x-y|}{2 N}\right)
$$

(independent of $\sigma$ and $\lambda$ ) and hence the proof of the theorem follows from Theorem 3.1.

\section{References}

[1] K. Ahmad and R. Kumar, Pointwise convergence of wavelet packet series, Atti Sem. Fis. Univ. Modena, 48 (2000), 107-120.

[2] B. Behera, Wavelet packets associated with nonuniform multiresolution analyses, J. Math. Anal. Appl., 328 (2007), 1237-1246.

[3] L. Carleson, On the convergence and growth of partial sums of Fourier series, Acta Math., 116 (1966), $135-195$.

[4] J.-P. Gabardo and M. Nashed, Nonuniform multiresolution analyses and spectral pairs, J. Funct. Anal., 158 (1998), 209-241.

[5] J.-P. Gabardo and M. Nashed, An analogue of Cohen's condition for nonuniform multiresolution analyses, in: A. Aldroubi, E. Lin (Eds.), Wavelets, Multiwavelets and Their Applications, in: Cont. Math., 216, Amer. Math. Soc., Providence, RI, (1998), 41-61.

[6] J.-P. Gabardo and X. Yu, Wavelets associated with nonuniform multiresolution analyses and one-dimensional spectral pairs, $J$. Math. Anal. Appl., 323 (2006), 798-817.

[7] E. Hernandez and G. Weiss, A First Course on Wavelets, CRC Press, New York, 1996.

[8] R. A. Hunt, On the convergence of Fourier series, in: Proceedings of the Conference on Orthogonal Expansions and their Continuous Analogues (D. T. Haimo. Ed.), Southern Illinois University Press, (1968), 235-255.

[9] S. E. Kelly, M. A. Kon and L. A. Raphael, Pointwise convergence of wavelet expansions, Bull. Amer. Math. Soc., 30 (1994), 87-94.

[10] S. E. Kelly, M. A. Kon and L. A. Raphael, Local convergence for wavelet expansions, J. Funct. Anal., 126 (1994), 102-138.

[11] S. G. Mallat, Multiresolution approximations and wavelet orthonormal bases in $L^{2}(\mathbb{R})$, Trans. Amer. Math. Soc., 315 (1989), $69-87$.

[12] Y. Meyer, Wavelets and Operators, Cambridge University Press, Cambridge, 1992.

[13] M. Nielsen, On convergence of wavelet packet expansions, Approx. Theory Appl. (N.S), 18 (2002), 34-50.

[14] M. Nielsen, Walsh-type wavelet packet expansions, Appl. Comput. Harmon. Anal., 9 (2000), $265-285$.

[15] G. G. Walter, Approximation of delta function by wavelets, J. Approx. Theory, 71 (1992), 329-343.

[16] G. G. Walter, Pointwise convergence for wavelet expansions, J. Approx. Theory, 80 (1995), 108-118. 